\title{
Topology of the world trade web
}

\author{
Ma Ángeles Serrano and Marián Boguñá \\ Departament de Física Fonamental, Universitat de Barcelona, Av. Diagonal 647, 08028 Barcelona, Spain
}

(Received 2 January 2003; published 11 July 2003)

\begin{abstract}
Economy, and consequently trade, is a fundamental part of human social organization which, until now, has not been studied within the network modeling framework. Here we present the first, to the best of our knowledge, empirical characterization of the world trade web, that is, the network built upon the trade relationships between different countries in the world. This network displays the typical properties of complex networks, namely, scale-free degree distribution, the small-world property, a high clustering coefficient, and, in addition, degree-degree correlation between different vertices. All these properties make the world trade web a complex network, which is far from being well described through a classical random network description.
\end{abstract}

DOI: 10.1103/PhysRevE.68.015101

PACS number(s): 89.75.Hc, 87.23.Ge, 05.70.Ln, 89.65.Gh

The world is facing a challenging era. Social, political, and economic arrangements initiated after the end of the Second World War are now culminating in the recognition of globalization, a process which has been accelerated by the new technological advances. When applied to the international economic order, globalization involves control of capital flow and liberalization of trade. As a consequence, economies around the world are becoming more and more interrelated, in other words, the world is becoming a global village $[1,2]$. In this scenario, trade plays a central role as one of the most important interaction channels between countries [3]. The relevance of the international trade system goes beyond the fundamental exchange of goods and services. For instance, it can also be the channel for crises spreading [4]. A good example is found in the recent Asiatic crisis, which shows how economic perturbations originating in a country can somehow propagate elsewhere in the world $[5,6]$. Thus, it seems natural to analyze the world trade system at a global level, every country being important, regardless of its size or wealth. Despite the extremely complex nature of the problem, relevant structural information can be extracted from modeling the system as a network, where countries are represented as vertices and trade channels as links between these vertices. In this way, the global trade system can be examined under a topological point of view. This analysis will reveal complex properties that cannot be explained by the classical random graph theory, first studied by Erdös and Rényi [7].

Complex networks have been the subject of an intense research activity over the last years $[8,9]$. Examples range from metabolic networks, where cell functionality is sustained by the network structure, to technological webs, where topology determines the system's ability to transmit information [10-13]. The term complex network typically refers to networks showing the following properties: (i) scale-free (SF) degree distribution, $P(k) \sim k^{-\gamma}$ with $2<\gamma$ $\leqslant 3$, where the degree $k$ is defined as the number of edges emanating from a vertex, (ii) the small-world property [14], which states that the average path length between any pair of vertices grows logarithmically with the system size; and (iii) a high clustering coefficient, that is, the neighbors of a given vertex are interconnected with high probability. In addition, degree-degree correlation has been recently added to this list since it appears as a common feature in many real-world networked systems $[12,15-17]$. This correlation accounts for the probability that a vertex of degree $k$ is connected to a vertex of degree $k^{\prime}$ and is a key issue for the correct description of the hierarchical organization within the network. This correlation is found to be assortative, that is, highly connected vertices tend to attach to other highly connected vertices, in social networks, such as scientific collaboration networks [15]; conversely, the correlation is disassortative in technological networks such as the Internet [12].

Among all the studied networks, the social and technological networks have become the paradigmatic examples of complex networks. Perhaps, the reason lies in the fact that each type exemplifies human activity working at one of two different levels: the cooperative level, where concepts, such as friendship, are dominant, and the competitive level, where the activity is governed by optimization criteria. These two different levels will probably lead to different attachment mechanisms which could be the origin of the assortative or disassortative degree-degree correlation observed in these networks. As an example of social network working at the competitive level, we study the network of trade relationships between different countries in the world, hereafter referred to as the world trade web (WTW). The topological characterization of the WTW is of primary interest for the modeling of crisis propagation at the global level as well as for the understanding of the effects that the new liberalist policies have on the world trade system. Moreover, we shall show that the WTW is a complex network sharing many properties with technological networks.

In order to perform our analysis, we extracted data from aggregated trade statistics tables in the International Trade Center site [19], which are based on the COMTRADE database of the United Nations Statistics Division. These tables contain, for each country, an import and an export list detailing the forty most important exchanged merchandises in the year 2000. Two major trading partners are also reported for each product. If we consider imports as in degrees and exports as out degrees, it is possible to construct a directed network where vertices represent countries and directed links represent the import and export relations between them. The fact that the number of merchandises is bounded is, a priori, a limitation for the analysis. However, it is possible to over- 
come this problem taking advantage of the symmetry between in and out degrees. Let $\widetilde{A}_{i j}^{i m p}$ and $\widetilde{A}_{i j}^{e x p}$ be the import by export adjacency matrices calculated from the import and export databases. Each adjacency matrix is defined so that $\widetilde{A}_{i j}=1$ if country $i$ imports from or exports to country $j$ and zero otherwise. These matrices account only for a subset out of the total number of actual connections between countries. The import and export connections that are of little relevance for a given country are not considered in matrices $\widetilde{A}$, although they may be relevant to the partners as the symmetric export and import links. In fact, import and export definitions can apply to the same trade flow depending on whether origin or destination is considered. This implies that the complete adjacency matrices satisfy the symmetry relation $A_{i j}^{i m p}$ $=A_{j i}^{e x p}$, which can be used in order to recover missing information from the original matrices. Thus, we can write

$$
A_{i j}^{i m p}=\frac{1}{1+\delta_{\left(\widetilde{A}_{i j}^{i m p}+\widetilde{A}_{j i}^{e x p}\right), 2}}\left[\widetilde{A}_{i j}^{i m p}+\widetilde{A}_{j i}^{e x p}\right],
$$

where $\delta_{,}$, is the Kronecker delta function. In this way we obtain an adjacency matrix where each connection is relevant, at least, to one of the two involved countries. At this point, it is worth noticing that we consider the unweighted version of the WTW. Since the weight of a link can be different, depending on the import or export point of view, it is unclear how these weights should be assigned. After this symmetrization procedure, we obtain a directed network with 179 vertices representing countries and 7510 directed links representing commercial channels among them. The average degree of this network is $\left\langle k_{\text {in }}\right\rangle=\left\langle k_{\text {out }}\right\rangle=30.9$.

The question that first arises refers to the directed nature of the WTW. In fact, the in and the out degrees of a given vertex are random quantities that may be correlated. A complete description should involve the knowledge of the joint probability $p\left(k_{\text {in }}, k_{\text {out }}\right)$. This function is often difficult to obtain although relevant information can be extracted from the correlation coefficient $r=\left(\left\langle k_{\text {in }} k_{\text {out }}\right\rangle-\langle k\rangle^{2}\right) / \sigma_{\text {in }} \sigma_{\text {out }}$, where $\sigma_{\text {in }}\left(\sigma_{\text {out }}\right)$ are the in (out) standard deviations (notice that $\left\langle k_{\text {in }}\right\rangle=\left\langle k_{\text {out }}\right\rangle=\langle k\rangle$ ). For the WTW this coefficient is $r$ $=0.91$, pointing to a strong similarity in the number of in and out connections. However, not all these connections run in both directions, that is, the fact that country $A$ imports from country $B$ does not necessary imply that country $B$ imports from country $A$. In order to quantify this effect we compute the reciprocity of the network, defined as the fraction of links pointing simultaneously to both ends of the link. In our case the reciprocity is 0.61 . These results suggest that actually the WTW may be thought of as an undirected network without losing relevant topological information (that is, considering all directed edges as undirected). The average degree for this definition of the undirected WTW is $\langle k\rangle$ $\approx 43$. This value is, indeed, very high if we take into account the size of the network, and, apparently, that could distort the analysis. An alternative way to define the undirected WTW is to consider only bidirectional edges. In this case, the average

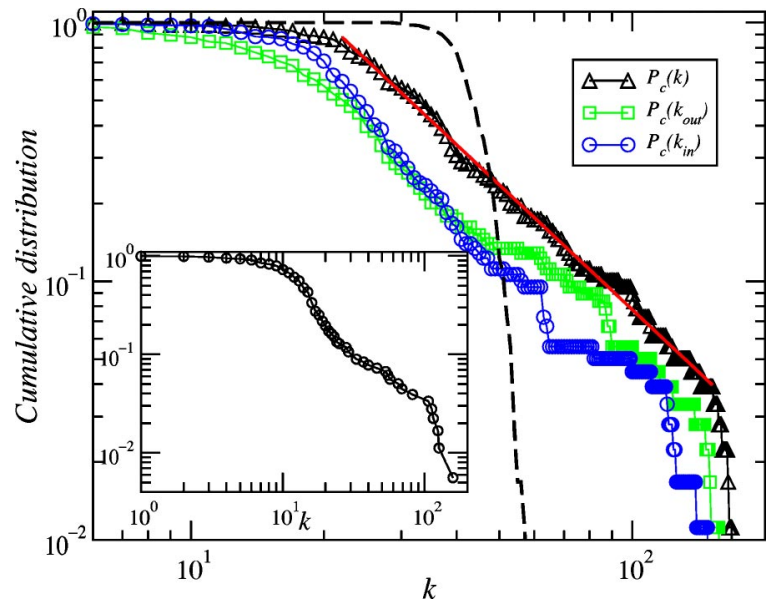

FIG. 1. Cumulative in- and out-degree distributions, $P_{c}\left(k_{i n}\right)$ and $P_{c}\left(k_{\text {out }}\right)$, and undirected $P_{c}(k)$ corresponding to the import and export world trade web. The solid line is a power law fit of the form $P_{c}(k) \sim k^{\gamma-1}$ with $\gamma=2.6 \pm 0.1$. The cumulative distribution of the equivalent random network with the same average degree is also shown. Inset: cumulative distribution for the WTW with reciprocal edges.

degree reduces significantly, $\langle k\rangle \approx 18$, although, as shown in the insets of the figures, the results remain basically unchanged.

One of the most important topological properties of a network is the degree distribution, $P(k)$. This quantity measures the probability of a randomly chosen vertex to have $k$ connections to other vertices. In our case, due to the directed nature of the network, we have to distinguish between inand out-degree distributions. Figure 1 shows the in, out, and undirected cumulative distributions for the WTW, defined as $P_{c}(k) \equiv \sum_{k^{\prime}=k} P\left(k^{\prime}\right)$. In all cases, the cumulative distribution shows a flat approach to the origin, indicating the presence of a maximum in $P(k)$, at $k \sim 20$, and, in this respect, similar to the Erdös-Rényi network. However, for $k>20$ the cumulative distribution is followed by a power law decay $P_{c}(k) \sim k^{1-\gamma}$, with $\gamma \approx 2.6$, showing a strong deviation from the exponential tail predicted by the classical random graph theory. The exponent $\gamma$ is found to be within the range defined by many other complex networks and, thus, we can state that the WTW belongs to the recently identified class of SF networks $[8,9]$. The SF property implies an extremely high level of degree heterogeneity. Indeed, the second moment of the degree distribution, $\left\langle k^{2}\right\rangle$, diverges in the thermodynamic limit for any SF network.

It may be surprising that the SF region does not extend to the whole degree domain and that, for small values of the degree, the distribution is similar to the Erdös-Rényi network. In fact, the underlying preferential attachment mechanisms [18] could differ depending on the particular political and economic situation of a country. Low-degree countries, most of which turn out to be the poorest, are basically constrained to subsistence trade flows and, therefore, preferential attachment mechanisms could not hold. As expected, there exists a positive correlation between the number of trade channels of a country and its wealth, measured by the per capita Gross Domestic Product (GDP) [19]. This correlation 


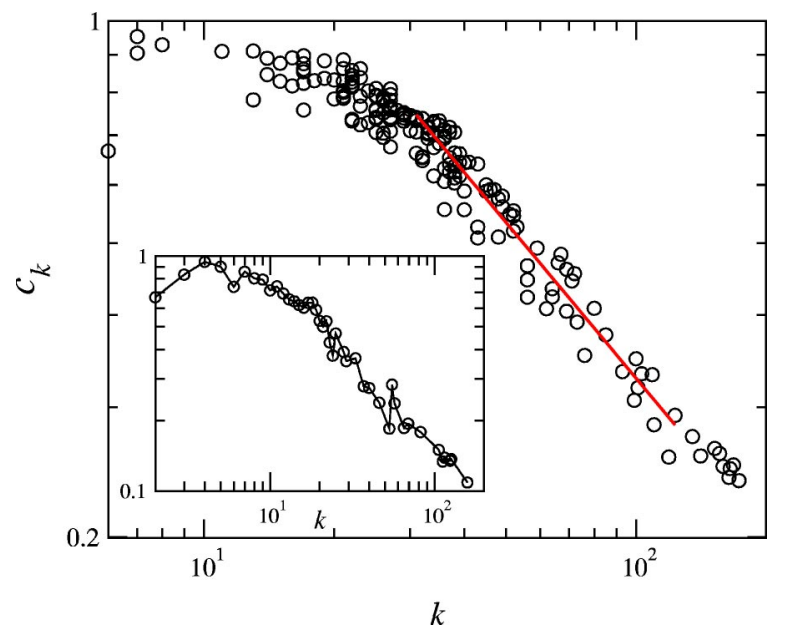

FIG. 2. Clustering coefficient of single countries as a function of their degree for the undirected version of the WTW. The solid line is a power law fit of form $c_{k} \sim k^{-\omega}$ with $\omega=0.7 \pm 0.05$. Inset: average clustering coefficient, as a function of the degree, for the WTW with reciprocal edges.

is found to be high, 0.65 , which means that, indeed, most low-connected countries are poor countries-Angola, Somalia, Rwanda, Cambodia....- and most high-connected countries are rich countries - the USA, Japan, Germany, and the UK, for example. However, there also exists a significant number of cases in the reversal situation, that is, high per capita GDP countries with a small number of connections and low per capita GDP countries with a large number of trade channels. A germane example for the first circumstance is that of Norway or Iceland, which are among the top ten wealthier countries but only have 56 and 24 trade channels, respectively. For the second case, Brazil, China, or Russia are typical examples.

Thanks to a number of recent studies, it is becoming more and more evident that real networks are not completely random but they are organized according to a hierarchical structure $[11,12,17,20]$. This hierarchy is usually analyzed by means of the clustering coefficient and the degree-degree correlation. The clustering coefficient of vertex $i$, of degree $k_{i}$, is defined as $c_{i} \equiv 2 n_{i} / k_{i}\left(k_{i}-1\right)$, where $n_{i}$ is the number of neighbors of $i$ that are interconnected. If hierarchy was not present in the system, $c_{i}$ should be a random quantity independent of any other property. Figure 2 shows the clustering coefficient of the undirected WTW as a function of the vertex's degree. As is clearly seen, this function has a strong dependence on the vertex's degree, with a power law behavior $c_{k} \sim k^{-\omega}$, with $\omega=0.7 \pm 0.05$. The clustering coefficient averaged over the whole network is $C=0.65$, greater by a factor 2.7 than the value corresponding to a random network of the same size.

Hierarchy is also reflected on the degree-degree correlation through the conditional probability $P\left(k \mid k^{\prime}\right)$, which measures the probability of a vertex of degree $k^{\prime}$ to be linked to a vertex of degree $k$. Again, this function is difficult to measure due to statistical fluctuations. In order to characterize this correlation, it is more useful to work with the average nearest neighbors degree (ANND), defined as $\left\langle k_{n n}(k)\right\rangle$

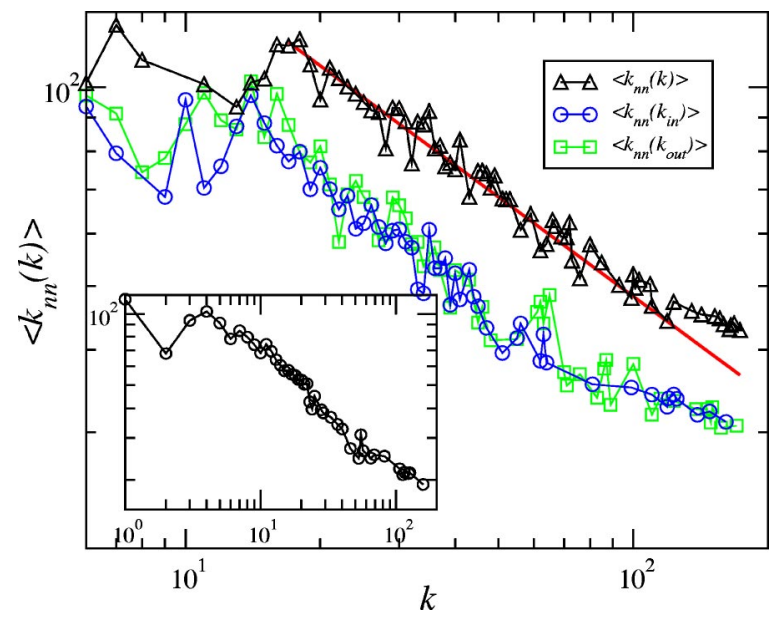

FIG. 3. Average in (out, undirected) nearest neighbors degree as a function of the in (out, undirected) degree of the vertex. The solid line is a fit of the form $\left\langle k_{n n}(k)\right\rangle \sim k^{-\nu_{k}}$ with $\nu_{k}=0.5 \pm 0.05$. Inset: the same for the WTW with reciprocal edges.

$\equiv \Sigma_{k^{\prime}} k^{\prime} P\left(k^{\prime} \mid k\right)[16]$. For uncorrelated networks, this function reads $\left\langle k_{n n}\right\rangle=\left\langle k^{2}\right\rangle /\langle k\rangle$, independent of $k$. However, recent studies have revealed that almost all real networks show degree-degree correlation $[12,15]$ which translates into a $k$ dependence in the ANND. This correlation can be assortative or disassortative depending on whether the ANND is an increasing or decreasing function of the degree. Figure 3 reports the ANND for the directed and undirected versions of the WTW. It can be observed a clear dependency on the vertex's degree, with a power law decay $\left\langle k_{n n}(k)\right\rangle \sim k^{-\nu_{k}}$ with $\nu_{k}=0.5 \pm 0.05$. This result means that the WTW is a disassortative network where highly connected vertices tends to connect to poorly connected vertices. This result, together with the scaling law $c_{k} \sim k^{-\omega}$ reveals a hierarchical architecture of highly interconnected countries that belong to influential areas which, in turn, connect to other influential areas through hubs.

Surprisingly, these results point to a high similarity between the WTW and the Internet. Indeed, the Internet is a SF network, with a critical exponent $\gamma=2.2$, which is also organized in a hierarchical fashion. The functional behavior found for the clustering coefficient and the ANND is a power law decay as a function of the degree with exponents $\omega_{\text {int }}$ $=0.75$ and $\nu_{k}=0.5$ [12], exponents that turn out to be very similar to the ones reported here for the WTW. In some sense, these results are not surprising since both are competitive systems evolving in a quasi free market and, in both cases, there exists, for instance, a geographic limitation that increases the connection costs and thus acts as a constraint in the optimization process of each vertex. Table I presents a summary of the main characteristics of the WTW, the Internet [12], and a random graph of the same size and average degree as the WTW. The average path length, defined as the average of the shortest distances between all pairs of vertices, is $\langle d\rangle=1.8$, which, in this case, is very similar to the corresponding random network of the same size and average degree.

In conclusion, this first approach to the topology of the 
TABLE I. Average degree $\langle k\rangle$, shortest path length $\langle d\rangle$, clustering coefficient $C$, exponent of degree distribution $\gamma$, exponent of clustering, $\omega$, and exponent of the average nearest neighbors degree $\nu_{k}$, for the world trade web (WTW), the Internet at the autonomous system level, and a random graph (RG) of the same size as the WTW.

\begin{tabular}{lccccccc}
\hline \hline & Size & $\langle k\rangle$ & $\langle d\rangle$ & $C$ & $\gamma$ & $\omega$ & $\nu_{k}$ \\
\hline WTW & 179 & 43 & 1.8 & 0.65 & 2.6 & 0.7 & 0.5 \\
Internet & 5287 & 3.8 & 3.7 & 0.24 & 2.2 & 0.75 & 0.5 \\
RG & 179 & 43 & 1.73 & 0.24 & - & 0 & 0 \\
\hline \hline
\end{tabular}

WTW points out some previously unnoticed features that are of primary importance in the understanding of the new international order. Over the last decades, international trade has grown faster than the world economy as a whole, increasing nation's economic integration. Our research suggests that this evolution is guided by collective phenomena, and that self-organization plays a crucial role in structuring the WTW scale-free inhomogeneities and its hierarchical architecture.
These results provide even more evidence that, due to the globalization process supported by the technological advances (which have removed many geographic, economic and technical constraints), the trade system has become a self-organized complex system that must be considered as a whole. It remains an open question if these properties could also be made apparent at other different scales, for instance, in the trade relations between regions, cities, or even individuals. At the country level, the activity is driven by competition. In the same way as it occurs with the Internet, optimization criteria are applied to local decisions made by the individual vertices. The findings in this paper may lead one to consider that there exist underlying mechanisms common to all competitive systems, characterized by disassortative associations, and such mechanisms may differ from the evolutionary processes in social cooperative networks, characterized by assortative associations.

Acknowledgements are due to Romualdo Pastor-Satorras for helpful discussions and advice. This work was partially supported by the European commission FET Open Project No. COSIN IST-2001-33555.
[1] P. Krugman, The Accidental Theorist, and other Dispatches from the Dismal Science (Norton, New York, 1998).

[2] S. Amin, Capitalism in the Age of Globalization: The Management of Contemporary Society (Zed Books, London, 1997).

[3] B.R. Koka, J.E. Prescott, and R. Madhavan, J. Int. Business Stud. 30, 127 (1999).

[4] R. Glick and A. Rose, J. Int. Money Fin. 18, 603 (1999).

[5] M. Noland, L-G Liu, S. Robinson, and Z. Wang, Global Economics Effects of the Asian Currency Devaluations (International Institute for Economics, Washington, D.C., 1998).

[6] M. Goldstein, The Asian Financial Crisis: Causes, Cures and Systemic Implications (International Institute for Economics, Washington, D.C., 1998).

[7] P. Erdös and P. Rényi, Publ. Math. (Debrecen) 6, 290-297 (1960); P. Erdös and P. Rényi, Publ. Math. Inst. Hung. Acad. Sci. 5, 17 (1960).

[8] R. Albert and A.-L. Barabási, Rev. Mod. Phys. 74, 47 (2002).

[9] S.N. Dorogovtsev and J.F.F. Mendes, Adv. Phys. 51, 1079 (2002)

[10] H. Jeong, B. Tombor, R. Albert, Z.N. Oltvai, and A.-L. Bara- bási, Nature (London) 407, 651 (2000).

[11] E. Ravasz, A.L. Somera, D.A. Mongru, Z.N. Oltvai, and A.-L. Barabási, Science 297, 1551 (2002).

[12] A. Vázquez, R. Pastor-Satorras, and A. Vespignani, Phys. Rev. E 65, 066130 (2002).

[13] R. Albert, H. Jeong, and A.-L. Barabási, Nature (London) 401, 130 (1999).

[14] D.J. Watts and S.H. Strogatz, Nature (London) 393, 440 (1998).

[15] M.E.J. Newman, Phys. Rev. Lett. 89, 208701 (2002).

[16] R. Pastor-Satorras, A. Vázquez, and A. Vespignani, Phys. Rev. Lett. 87, 258701 (2001).

[17] K.-I. Goh, B. Kahng, and D. Kim, Phys. Rev. Lett. 88, 108701 (2002).

[18] A.-L. Barabási and R. Albert, Science 286, 509 (1999).

[19] Import and Export databases are publicly avalable at the address http://www.intracen.org/menus/countries.htm and the gross domestic product at http://www.tswoam.co.uk/world

[20] E. Ravasz and A.-L. Barabási, Phys. Rev. E 67, 026112 (2003). 